\title{
EL ROL DEL CONOCIMIENTO EN EL CRECIMIENTO ECONÓMICO: UN ANÁLISIS ESPACIAL PARA MÉXICO
}

\author{
Gabriela Sánchez Trujillo, ${ }^{a}$ Saúl Basurto Hernández ${ }^{\mathrm{b}}$ \\ y Sandra Galván Vargas ${ }^{c}$
}

Fecha de recepción: 30 de septiembre de 2019. Fecha de aceptación: 2 de febrero de 2020.

$$
\text { https://doi.org/10.22201/iiec.20078951e.2020.202.69483 }
$$

\begin{abstract}
Resumen. El objetivo de la presente investigación es el análisis de la influencia del nivel educativo de la fuerza laboral, la acumulación del capital intelectual, la capacidad de innovación y de la transferencia de conocimientos sobre la actividad económica de las entidades federativas de México. Para lograr este objetivo, se estimaron diferentes especificaciones de un modelo panel que toman en cuenta los patrones de correlación espacial de la actividad económica y de argumentos no observables de la función de producción de entidades colindantes. Los resultados sugieren que, al disminuir la proporción de trabajadores con niveles de educación más bajos, al aumentar la acumulación de capital intelectual y al intensificar el intercambio de bienes y servicios con el exterior, el nivel de producción se incrementa.
\end{abstract}

Palabras clave: economía del conocimiento; capital humano; entidades federativas; estimaciones regionales; modelo de panel.

Clasificación JEL: C23; C33; D83; E24; R11.

\section{THE ROLE OF KNOWLEDGE IN ECONOMIC GROWTH: a SPATIAL ANALYSIS FOR MEXICO}

\begin{abstract}
The aim of this research is to analyze the impact of the education level of the labor force, the accumulation of intellectual capital, the capacity for innovation, and the transfer of knowledge on the economic activity of Mexico's federal entities. To achieve this goal, various specifications for a panel model were calculated that take into account the spatial correlation patterns of economic activity and the unobservable claims of neighboring entities' production functions. The results suggest that by decreasing the proportion of workers with lower education levels, increasing the accumulation of intellectual capital, and intensifying the exchange of goods and services abroad, the production level increases.
\end{abstract}

Key Words: knowledge-driven economy; human capital; federal entities; regional calculations; panel model.

${ }^{a}$ Universidad Autónoma del Estado de Hidalgo (UAEH), México; ${ }^{b}$ Universidad Nacional Autónoma de México (unam), Facultad de Economía, México; ${ }^{\mathrm{C}}$ Universidad Autónoma de Barcelona (UAB), España. Correos electrónicos: magdags@uaeh.edu.mx; sbasurto@economia.unam.mx; sandragv06@gmail.com, respectivamente. 


\section{INTRODUCCIÓN}

En las últimas décadas la integración de las economías se intensificó como resultado de los procesos de globalización. Dicha integración permite un flujo mayor de mercancías, servicios, recursos financieros y conocimientos. La teoría económica, especialmente la teoría del crecimiento endógeno, argumenta que la creación y la transferencia de conocimientos tienen un efecto positivo sobre el producto (Lucas, 1988; Romer, 1990). Este fenómeno no sólo se presenta en el lugar en el que se crea el conocimiento mismo, sino también en las economías y regiones que interactúan con los generadores de dichos conocimientos (Lucas, 1988). Dado el argumento anterior, esta investigación tiene como objetivo central identificar la relación que existe entre diversas formas de conocimiento y la actividad económica estatal.

Hasta el momento, la literatura especializada indica que existen diferentes formas de conocimiento. Las economías cuentan con un stock o acumulación de conocimientos que se materializan en cantidad y calidad del capital físico (Lucas, 1988). Asimismo, existe una acumulación de conocimientos en la fuerza de trabajo con la que cuenta una región, es decir, el nivel de capital humano. Becker (1994) indica que la "revolución del capital humano" inició con los estudios de Schultz (1961), Mincer (1958), Rosen (1976), entre otros. También sugiere que la educación y la capacitación fuera de la escuela son las dos formas de inversión en capital humano más importantes y que aquellas regiones que muestran un crecimiento económico persistente, suelen ligarse a grandes incrementos en el nivel de educación y de capacitación de su fuerza laboral. Por su parte, Coleman (1988) argumenta que el capital social dentro del núcleo familiar (relación entre padres e hijos) tiene una influencia importante sobre el nivel de escolaridad de los hijos.

Teece (1998) indica que la generación de nuevos conocimientos se puede materializar en la acumulación de capital intelectual, por ejemplo, las patentes o derechos de autor. Este tipo de conocimientos suelen modificar la eficiencia con la que se utilizan los factores de producción. Sin embargo, se puede excluir a agentes económicos mediante los derechos de propiedad.

Otros autores, como Arrow (1971), Lucas (1988) y Houghton y Sheehan (2000) argumentan que el conocimiento proviene de un proceso de aprendizaje -conocido como learning-by-doing-, a través de la experiencia. Dicho aprendizaje suele asociarse con incrementos de productividad asociados a los aumentos en exportaciones de bienes que no eran producidos originalmente; por ejemplo, los "milagros de crecimiento" en Corea, Taiwán, Hong Kong y 
Singapur. Lucas (1988) explica que estas economías materializaron su experiencia (learning-by-doing) en un aumento de productividad en sectores en los cuales no existía un alto nivel de especialización.

Un incremento en el nivel de conocimiento con el que cuenta una economía puede generarse a través de diferentes vías. El stock de talento depende de los gastos en investigación y desarrollo $(\mathrm{R} \& \mathrm{D})$ que lleven a cabo los diversos agentes económicos, quienes también tienen la posibilidad de adquirir conocimientos generados en otros lugares, por ejemplo, la compra de maquinaria de alta tecnología para lograr una mayor eficiencia en el uso de insumos del proceso de producción (capital físico).

Respecto al capital intangible, la compra de licencias o derechos de uso de patentes representan un mecanismo adicional para incrementar el stock de conocimientos y, por ende, la eficiencia en el uso de los factores de producción. De la misma manera, la educación y capacitación permiten contar con capital humano mejor calificado.

Para identificar la influencia que tiene la acumulación de conocimientos en el crecimiento económico, este trabajo estima diferentes especificaciones de una función de producción tipo Cobb-Douglas. Las especificaciones comprenden nueve modelos de datos panel que involucran el uso de efectos fijos, aleatorios, dinámicos y de correlación espacial.

Los resultados sugieren que la calificación de la fuerza de trabajo contribuye de manera significativa al producto estatal; niveles inferiores de educación de la fuerza de trabajo se asocian con un crecimiento económico menor; los parámetros estimados asociados al número de investigadores que pertenecen al Sistema Nacional de Investigadores ( $\mathrm{SNI}$ ) no son estadísticamente significativos en la mayoría de las especificaciones; el capital intangible muestra una asociación positiva (limitada) con el nivel de producto; los canales de difusión de conocimiento también juegan un papel importante en el nivel de producción; contrario a las expectativas iniciales, las estimaciones sugieren que un incremento en el monto de la Inversión Extranjera Directa (IED) mantiene una asociación negativa con el Producto Interno Bruto (PIB), en particular, en el sur de México; y, el grado de apertura comercial o tamaño del comercio con el exterior se asocia positivamente con el nivel de producción.

La parte restante de este documento de investigación se estructuró de la siguiente manera: se presenta una revisión de estudios empíricos que justifica la selección de la metodología utilizada para responder a la pregunta de investigación. También se muestra la metodología de modelos panel utilizados para identificar la influencia de las diferentes formas de conocimiento en el nivel 
de producción. Después, se describe el conjunto de materiales utilizados en el análisis. Para luego presentar los resultados del conjunto de modelos panel estimados. Posteriormente, se presenta una breve discusión sobre los resultados obtenidos. Y, por último, se comentan una serie de conclusiones.

\section{REVISIÓN DE LITERATURA: ESTUDIOS EMPÍRICOS}

Para identificar la frontera del conocimiento y seleccionar la metodología adecuada, se llevó a cabo una revisión sistemática de la literatura sobre estudios empíricos. Al analizar el conjunto de estudios, se observa que esta rama de la literatura centra su atención en países pertenecientes a la Organización para la Cooperación y Desarrollo Económicos (OCDE), como son los casos de Coe y Helpman (1995), Engelbrecht (1997), Kao et al. (1999), Frantzen (2000), Guellec y De La Potterie (2002) o Zhu y Jeon (2007). Los autores argumentan que el capital humano afecta la productividad total de los factores, dado que, se considera como un vehículo importante de transferencia de conocimientos. Este fenómeno se origina debido a la relación directa que existe entre el nivel de capital humano y su capacidad para aprovechar los aumentos de productividad en otros países o regiones con las que se tiene un alto grado de vinculación (Nursamsu y Hastiadi, 2015).

Autores como Basant y Fikkert (1996) y Coe et al. (1997) indican, por su parte, que la transferencia de conocimientos mediante la adquisición de tecnologías existentes tiene un efecto positivo sobre el nivel de producción. Respecto al papel que desempeńa la IED en el impulso de la actividad económica, autores como Coe y Helpman (1995) y Guellec y De La Potterie (2002) reconocen que, si dicha inversión se orienta hacia la innovación, existe una asociación positiva entre la IED y el PIB. Coe y Helpman (1995) definen que dichos efectos se potencian cuando aumenta el grado de apertura comercial. Sin embargo, otros autores como Kao et al. (1999) y Mendoza-Cota (2011) indican que la IED no influye en el nivel de producción cuando dicha inversión no se dirige hacia lograr una mayor eficiencia en el uso de insumos.

La literatura sugiere el uso de diversas metodologías para identificar la relación entre las formas de conocimiento y el nivel del producto. En este conjunto destacan los modelos panel de datos agrupados (POLS), efectos fijos $(\mathrm{FE})$, efectos aleatorios (RE), efectos dinámicos (GMM) y de correlación espacial. Respecto a estos últimos, Naveed y Ahmad (2016) utilizan modelos es- 
paciales auto-regresivos (SAR) y Durbin (SDM) identificando efectos indirectos de la tecnología y del conocimiento en los niveles de producción de regiones colindantes. Esto implica que la forma en la que el conocimiento influye en la actividad económica se diluya en el espacio.

Con base en la revisión de estudios empíricos y en las restricciones que impone la disponibilidad de información estadística, se analiza sólo la influencia de algunas formas de conocimiento sobre la actividad económica de las entidades federativas de México. Este conjunto incluye: el nivel de escolaridad de la fuerza de trabajo, la acumulación de capital intelectual, la capacidad de innovación y la transferencia de conocimientos y experiencia vía intercambio de bienes y servicios.

Algunos medios de conocimiento identificados en la revisión de literatura no son directamente observables, como las diferentes maneras de capital social y su influencia sobre la formación de capital humano, la capacitación de los trabajadores o todos los procesos de learning-by-doing. Estas formas de conocimiento son observables a nivel microeconómico, e.g. empresas, pero están fuera del objetivo de este trabajo (se puede consultar a Basurto y Sánchez, 2020, al respecto).

\section{METODOLOGÍA}

Para identificar la relación entre conocimiento y nivel de producción se utilizan modelos panel. De acuerdo con Cameron y Trivedi (2005), un modelo panel, en su forma más simple, puede estimarse como un modelo pols, el cual se define de la siguiente manera:

$$
y_{i t}=\alpha+X_{i t}^{\prime} \beta+u_{i t}
$$

donde $y_{i t}$ es el nivel de producción del estado $i$ en el año $t, \alpha$ denota un término constante, $X_{i t}^{\prime}$ contiene el conjunto de variables explicativas del producto en la entidad federativa $i$ en el año $t$, el vector $\beta$ contiene los parámetros asociados a cada una de las variables explicativas y $u_{i t}$ es el término de error.

Para el caso del modelo pols, se asume que las variables explicativas son exógenas y no existe correlación de éstas con el término de error, esto es, $E\left(u_{i t} \mid X_{i t}^{\prime}\right)=0$, donde $u_{i t}=\alpha_{i}+\varepsilon_{i t}$. Si existe correlación entre el término de error, $u_{i t}$, y las variables explicativas, $X_{i t}^{\prime}$, los parámetros estimados, $\hat{\beta}$, 
en la ecuación (1) mostrarán sesgos. Cuando esto ocurre, se pueden utilizar modelos panel de FE O RE. En el primero se asume que existe correlación entre los efectos individuales y las variables explicativas y que no existe correlación entre el término de error, los efectos individuales y el conjunto de variables explicativas. De esta forma, se tiene que $E\left(\alpha_{i} \mid X_{i t}^{\prime}\right) \neq 0$ y $E\left(\varepsilon_{i t} \mid \alpha_{i}, X_{i t}^{\prime}\right)=0$. En el segundo caso se asume que el componente de efectos individuales, $\alpha_{i}$, es puramente aleatorio y que no existe correlación entre dichos efectos y las variables explicativas. De esta manera, se espera que $E\left(\alpha_{i} \mid X_{i t}^{\prime}\right)=0$ y que $E\left(\varepsilon_{i t} \mid \alpha_{i}, X_{i t}^{\prime}\right)=0$.

Para discriminar entre los modelos pOLs, FE y RE se utiliza la prueba de Hausman (Hausman, 1978), que compara los parámetros estimados en los modelos FE y RE, $\hat{\beta}_{F E}$ y $\hat{\beta}_{R E}$, para identificar si las diferencias entre dichos parámetros son estadísticamente significativas o no. Si los efectos individuales son fijos, entonces los parámetros estimados en los modelos POLS y RE son inconsistentes y, por lo tanto, se prefiere el modelo FE.

El estadístico de la prueba de Hausman ${ }^{1}$ se calcula a partir de las diferencias entre los parámetros $\hat{\beta}_{F E}$ y $\hat{\beta}_{R E}$, ponderados por la varianza y se define de la siguiente manera:

$$
H=\left(\hat{\beta}_{F E}-\hat{\beta}_{R E}\right)^{\prime}\left[\operatorname{Var}\left(\hat{\beta}_{F E}\right)-\operatorname{Var}\left(\hat{\beta}_{R E}\right)\right]^{-1}\left(\hat{\beta}_{F E}-\hat{\beta}_{R E}\right)
$$

Con el propósito de incorporar elementos dinámicos de las variables explicativas, se estima un modelo panel a través del Método General de Momentos (Generalized Moments Method, GMM, por sus siglas en inglés), donde los rezagos de las variables explicativas se utilizan como instrumentos de la ecuación principal.

Para completar el análisis, se estiman diferentes especificaciones de modelos panel FE y RE que toman en cuenta patrones de correlación espacial. El modelo espacial auto-regresivo (SAR) asume que el nivel de actividad económica del estado $i$ se asocia con el nivel de actividad económica de los estados colindantes $j$ y se define como:

1 La hipótesis nula en la prueba de Hausman plantea que no existen diferencias significativas entre $\hat{\beta}_{F E}$ y $\hat{\beta}_{R E}$, esto es, $H_{0}: \hat{\beta}_{F E}=\hat{\beta}_{R E}$, pues ambos estimadores son consistentes y los efectos individuales son puramente aleatorios. Por otro lado, la hipótesis alternativa sugiere que existen diferencias significativas entre $\hat{\beta}_{F E}$ y $\hat{\beta}_{R E}$, es decir $H_{1}: \hat{\beta}_{F E} \neq \hat{\beta}_{R E}$, debido a que $E\left(\alpha_{i} \mid X_{i t}^{\prime}\right) \neq 0$. 


$$
y_{i t}=\rho W y_{i t}+X_{i t}^{\prime} \beta+\alpha_{i}+\varepsilon_{i t}
$$

donde, $W$ es una matriz de dimensiones $i * i$ y con entradas $w_{i j} \in W$, las cuales indican el peso asociado, o proximidad, a cada elemento $i, j$. Los elementos de la diagonal en la matriz $W, w_{i i}$, se les asigna el valor de 0 para excluir la contradicción de que un estado colinde consigo mismo. El parámetro $\rho$ indica el grado de asociación entre el nivel de actividad económica en el estado $i$ y los estados vecinos $j$. $^{2}$

Las especificaciones anteriores asumen que no existe correlación espacial entre los términos de error. En algunos casos, variables relevantes que no son observables forman parte del término de error, e.g. capital físico o capital natural. Para corroborar que las variables omitidas no causen algún sesgo en los parámetros estimados, el modelo de autocorrelación espacial (SAC) asume que existe correlación espacial entre los términos de error. De esta forma, el modelo saC supone que $\varepsilon_{i t}=\lambda W \varepsilon_{i t}+v_{i t}$, donde, $\lambda$ mide la correlación entre los errores espacialmente rezagados, $\varepsilon_{i t}$, y $v_{i t}$ es el término de error y sigue una distribución i.i.d. sobre los componentes de individuos y de tiempo.

De la expresión (3) se desprende un caso específico. Cuando se asume que $\rho=0$ y $\varepsilon_{i t}=\lambda W \varepsilon_{i t}+v_{i t}$ se puede estimar un modelo de correlación espacial de errores (sEM) (Anselin, 2013).

\section{MATERIALES}

Para estimar el conjunto de modelos definidos en la sección anterior, se utilizó información estadística de las 32 entidades federativas de México durante el periodo 2007-2016. La variable dependiente, $y_{i t}$, se define como el PIB estatal medido en precios de 2013.

Por motivos de presentación, la figura 1.1 muestra el PIB per cápita promedio anual del periodo. Se puede observar cierto grado de correlación entre el nivel de actividad económica de diferentes entidades federativas, por ejemplo, el dinamismo económico en la frontera norte de México parece impulsar el nivel de producción en estados como: Nuevo León, Sonora, Coahuila, Chihuahua y Tamaulipas. Por otro lado, el sur y algunos estados del centro del país se caracterizan por ser regiones con bajo nivel de actividad económica.

2 Nótese que, cuando $\rho=0$, el modelo SAR se reduce a alguno de los modelos POLS, FE o RE dependiendo del resultado de la prueba de Hausman. 
Respecto a la fuerza de trabajo, se utiliza la Población Económicamente Activa (PEA) por entidad federativa. La Secretaría del Trabajo y Previsión Social (sTPs) publica anualmente el número de personas que conforman la PEA en cada uno de los estados durante el periodo 2005-2017. Dado que el nivel de educación de los trabajadores juega un papel importante en la adaptación de conocimiento a los procesos de producción existentes, en la transferencia de conocimientos y en la generación de nuevos conocimientos que impulsan la actividad económica, se utiliza la PEA por nivel de instrucción en el conjunto de estimaciones. Las figuras 1.2-1.5 muestran el promedio de la proporción de la PEA que cuenta con educación primaria, secundaria, media y superior, respectivamente. En algunos casos se observa cierto grado de correlación espacial entre el PIB y el grado de calificación de la PEA, por ejemplo, los estados que colindan con la frontera norte y la Ciudad de México donde se observan niveles más altos de producción y trabajadores con educación superior.

Para México, el Instituto Nacional de Estadística y Geografía (INEGI) publica anualmente el monto de formación bruta de capital fijo a nivel nacional, sin embargo, dicha información no se encuentra disponible a nivel de entidad federativa. A pesar de los esfuerzos del instituto por construir dicho indicador para las entidades con base en los censos económicos, sólo se encuentra disponible para los años 2003, 2008 y 2013, lo que limita el análisis. Por lo tanto, en este trabajo de investigación se utilizó la formación bruta de capital fijo de los gobiernos estatales en el periodo 2003-2016 como una variable proxy de la formación de capital físico (véase figura 1.6).

La capacidad de las entidades federativas para generar nuevos conocimientos y su relación con el nivel de producción, se aproxima mediante el número de investigadores que pertenecen al sNI del Consejo Nacional de Ciencia y Tecnología (CONACYT). El consejo publica una serie anual del número total de investigadores pertenecientes al SNI en el periodo 2005-2017. Con base en dicha información estadística, se calcula el número de investigadores SNI por cada 100 mil habitantes. La figura 2.1 muestra el promedio de investigadores sNI durante dicho periodo.

Siguiendo la revisión de la literatura, el número de patentes puede ser utilizado como proxy del capital intelectual de alguna región o economía. El Instituto Mexicano de la Propiedad Industrial (IMPI) publica también una serie anual con el número de patentes por entidad federativa en el periodo 2003-2017. Para homogeneizar el capital intelectual, se utiliza el número de 
patentes por cada 100 mil habitantes. La figura 2.2 muestra la distribución geográfica del promedio de patentes durante el periodo de análisis.

El conjunto de estimaciones utiliza la IED como porcentaje del PIB para aproximar el grado de transmisión de conocimiento generado en otros países hacia las economías locales. El INEGI publica una serie anual de la IED por estado durante el periodo 2003-2017. La figura 2.3 muestra la distribución espacial del promedio de IED como porcentaje del PIB en el periodo 20032017. Los flujos de IED parecen alojarse en entidades federativas con niveles de capital humano, de capacidad de generar o adaptar nuevos conocimientos $\mathrm{y}$ de ingresos superiores a los de otros estados.

La intensidad de las interacciones entre dos economías se mide a través de la proporción de exportaciones e importaciones sobre el PIB, considerada como una variable proxy del grado de apertura comercial. El INEGI da a conocer el valor de las exportaciones por entidad federativa para el periodo 20072016, sin embargo, el valor de las importaciones no se encuentra disponible a nivel de entidades federativas.

De acuerdo con la figura 2.4, los estados del sur muestran los niveles más bajos en la intensidad de intercambio de bienes y servicios con otros países, sobre todo con Estados Unidos, debido a los altos costos de transporte de mercancías, mientras que los estados del norte suelen estar más vinculados con el mercado norteamericano.

Sujeta a la disponibilidad de información, se construyó una base de datos de tipo panel para identificar la influencia de diversos tipos de conocimiento en el nivel de producción de las entidades federativas que contiene información estadística completa para las variables ya descritas para el periodo 20072016. 



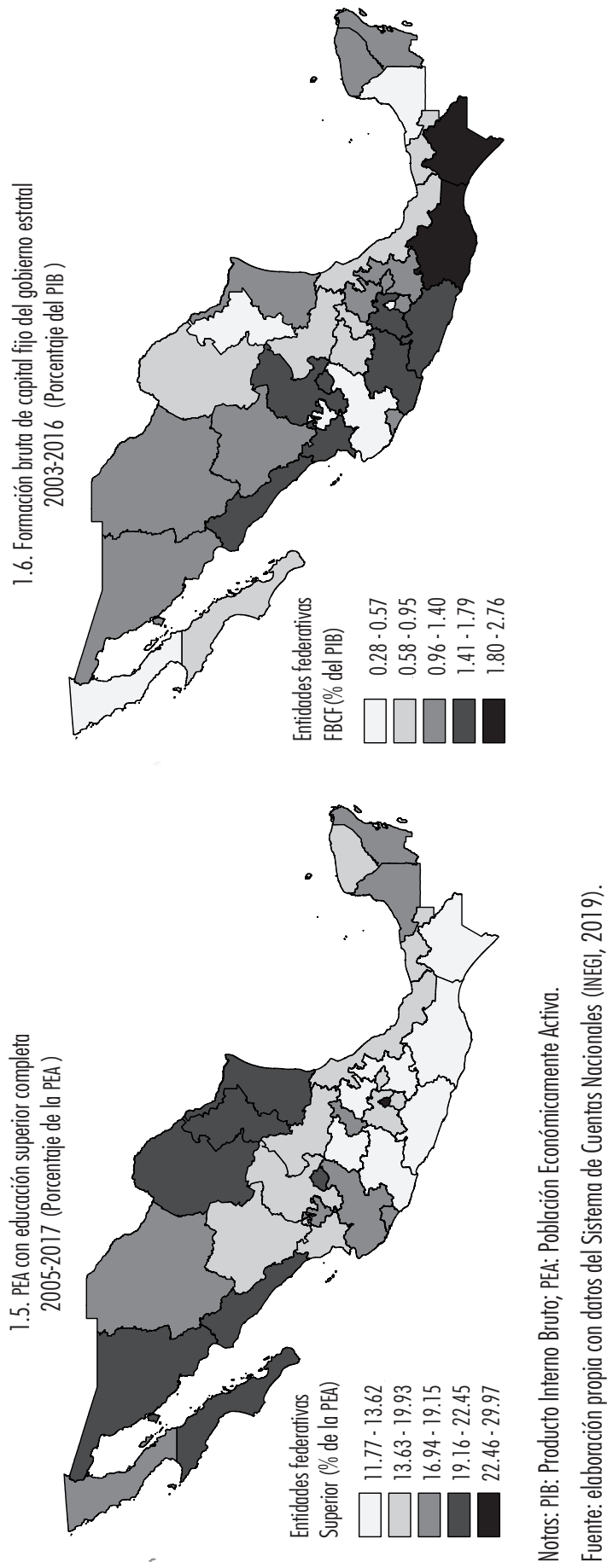

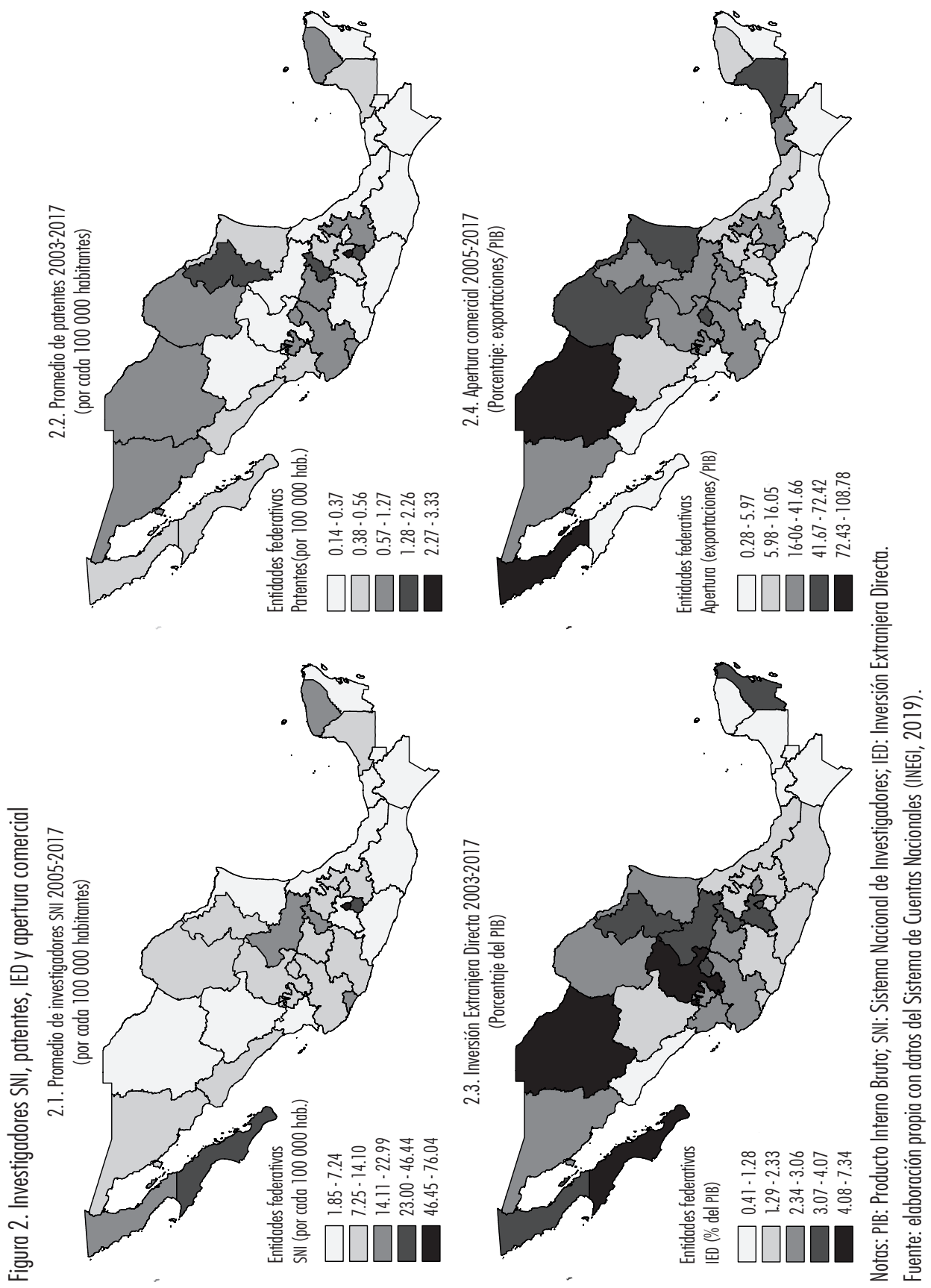


\section{RESULTADOS}

El conjunto de estimaciones incluye los siguientes modelos: POLS, FE, RE, GMM, SAR-FE, SAR-RE, SEM-FE, SEM-RE, y SAC-FE. En todos los casos, se utiliza el logaritmo de las variables. El cuadro 1 presenta los estadísticos de la prueba de Hausman, que sugiere que el modelo FE se prefiere sobre las especificaciones POLS y RE. Asimismo, la prueba de Hausman indica que se pueden descartar los modelos SAR-EA y SEM-EA. Por lo tanto, se puede centrar la atención en los parámetros estimados en los modelos FE, GMM, SAR-FE, SEM-FE Y SAC-FE. ${ }^{3}$

Cuadro 1. Pruebas de correcta especificación del modelo panel

\begin{tabular}{|c|c|c|c|c|}
\hline Comparación de modelos & Prueba & Chi-2 & Prob>Chi-2 & Resultado \\
\hline FE vs. RE & Hausman & 42.96 & 0.00 & $\mathrm{FE}$ \\
\hline SAR-FE vs. SAR-RE & Hausman & 27.77 & 0.01 & SAR-FE \\
\hline SEM-FE vs. SEM-RE & Hausman & 21.84 & 0.04 & SEM-FE \\
\hline
\end{tabular}

Fuente: elaboración propia con base en las estimaciones.

El cuadro 2 muestra los parámetros estimados para cada una de las especificaciones del modelo panel. De acuerdo con los parámetros estimados del modelo $\mathrm{FE}$, la fuerza de trabajo contribuye con $27.61 \%$ del valor de la producción total. El esfuerzo de los gobiernos estatales por incrementar el capital físico en las entidades parece no tener un impacto significativo sobre la producción. Este resultado puede deberse a que la magnitud de dichas inversiones (entre 0.28 y $2.76 \%$ del PIB) no es suficiente para impulsar el crecimiento económico. 
Gabriela Sánchez, Saúl Basurto y Sandra Galván

Cuadro 2. Parámetros estimados de los modelos panel

\begin{tabular}{|c|c|c|c|c|c|}
\hline Variables & $\begin{array}{l}\text { FE } \\
P I B\end{array}$ & $\begin{array}{c}\text { GMM } \\
P I B\end{array}$ & $\begin{array}{c}\text { SAR-FE } \\
\text { PIB }\end{array}$ & $\begin{array}{c}\text { SEM-FE } \\
P I B\end{array}$ & $\begin{array}{c}\text { SAC-FE } \\
P I B\end{array}$ \\
\hline \multirow[t]{2}{*}{ pea } & $0.2761^{\star \star}$ & $0.4778^{\star \star \star}$ & $0.2588^{\star \star}$ & $0.3875^{\star \star \star}$ & $0.1094^{\star}$ \\
\hline & $(0.1116)$ & $(0.1779)$ & $(0.1011)$ & $(0.1070)$ & $(0.0652)$ \\
\hline \multirow[t]{2}{*}{ pea ${ }^{\star}$ pea con educación superior (\%) } & 0.0021 & $0.1124^{\star \star \star}$ & 0.0005 & 0.0002 & 0.0036 \\
\hline & $(0.0054)$ & $(0.0178)$ & $(0.0049)$ & $(0.0050)$ & $(0.0032)$ \\
\hline \multirow[t]{2}{*}{ pea pea con educación media superior (\%) } & 0.0011 & -0.0081 & 0.0004 & 0.0018 & $0.0096^{\star \star \star}$ \\
\hline & $(0.0036)$ & $(0.0067)$ & $(0.0033)$ & $(0.0033)$ & $(0.0026)$ \\
\hline \multirow[t]{2}{*}{ pea $^{\star}$ pea con secundaria terminada (\%) } & 0.0019 & 0.0053 & -0.0011 & -0.0047 & $0.0215^{\star \star \star}$ \\
\hline & $(0.0070)$ & $(0.0209)$ & $(0.0064)$ & $(0.0067)$ & $(0.0042)$ \\
\hline \multirow[t]{2}{*}{ pea $^{\star}$ pea con secundaria incompleta (\%) } & $-0.0049^{\star \star}$ & $0.0152^{\star \star}$ & $-0.0043^{\star \star}$ & $-0.0049 \star \star$ & $-0.0044^{\star \star \star}$ \\
\hline & $(0.0021)$ & $(0.0060)$ & $(0.0019)$ & $(0.0019)$ & $(0.0014)$ \\
\hline \multirow[t]{2}{*}{ pea $^{\star}$ pea con estudios de primaria (\%) } & $-0.0195^{\star \star \star}$ & -0.0231 & $-0.0156^{\star \star \star}$ & $-0.0183^{\star \star \star}$ & -0.0052 \\
\hline & $(0.0059)$ & $(0.0186)$ & $(0.0054)$ & $(0.0057)$ & $(0.0032)$ \\
\hline \multirow[t]{2}{*}{ formación bruta de capital fijo del gobierno } & -0.0011 & $0.1116^{\star \star \star}$ & 0.0036 & 0.0073 & $0.0106^{\star \star}$ \\
\hline & $(0.0082)$ & $(0.0389)$ & $(0.0075)$ & $(0.0079)$ & $(0.0048)$ \\
\hline \multirow[t]{2}{*}{ sni (por cada 100000 hab.) } & 0.0402 & 0.0209 & 0.0325 & $0.0492^{\star \star}$ & -0.0204 \\
\hline & $(0.0258)$ & $(0.0350)$ & $(0.0234)$ & $(0.0240)$ & $(0.0157)$ \\
\hline \multirow[t]{2}{*}{ patentes (por cada 100000 hab.) } & $0.0098^{\star}$ & 0.0155 & $0.0096^{\star}$ & $0.0098^{\star \star}$ & 0.0037 \\
\hline & $(0.0055)$ & $(0.0275)$ & $(0.0050)$ & $(0.0049)$ & $(0.0035)$ \\
\hline \multirow[t]{2}{*}{ ied (\% del PIB) } & $-0.0064^{\star}$ & $-0.1654^{\star \star \star}$ & $-0.0066^{\star \star}$ & $-0.0079 \star \star$ & $-0.0066^{\star \star \star}$ \\
\hline & $(0.0037)$ & $(0.0256)$ & $(0.0034)$ & $(0.0034)$ & $(0.0025)$ \\
\hline \multirow[t]{2}{*}{ apertura comercial (exportaciones/PIB) } & 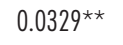 & $0.1197^{\star \star \star}$ & $0.0209^{*}$ & 0.0169 & $0.0433^{\star \star \star}$ \\
\hline & $(0.0134)$ & $(0.0158)$ & $(0.0125)$ & $(0.0130)$ & $(0.0088)$ \\
\hline \multicolumn{6}{|l|}{ Correlación espacial } \\
\hline \multirow[t]{2}{*}{ Rho $(\rho)$} & & & $0.2289 * \star \star$ & & $0.5216^{\star \star \star}$ \\
\hline & & & $(0.0606)$ & & $(0.0584)$ \\
\hline \multirow[t]{2}{*}{ Lambda $(\lambda)$} & & & & $0.2610^{\star \star \star}$ & $-0.9387^{\star \star \star}$ \\
\hline & & & & $(0.0674)$ & $(0.0621)$ \\
\hline
\end{tabular}




\begin{tabular}{lccccc}
\hline Variables & FE & GMM & SAR-FE & SEM-FE & SAC-FE \\
& PIB & PIB & PIB & PIB & PIB \\
\hline Varianza & \multicolumn{6}{c}{$0.0023^{\star \star \star}$} & $0.0023^{\star \star \star}$ & $0.0012^{\star \star \star}$ \\
\hline Sigma2_e & & & $(0.0002)$ & $(0.0002)$ & $(0.0001)$ \\
\hline Constante & $9.5227^{\star \star \star}$ & $1.2732^{\star \star \star}$ & & & \\
& $(1.4350)$ & $(0.4441)$ & & & \\
\hline Observaciones & 320 & 288 & 320 & 320 & 320 \\
R-cuadrada & 0.67 & 0.84 & 0.64 & 0.74 & 0.42 \\
Número de entidades & 32 & 32 & 32 & 32 & 32 \\
\hline
\end{tabular}

Notas: errores estándar robustos entre paréntesis; ${ }^{* \star \star} p<0.01 ;{ }^{\star \star} p<0.05 ;{ }^{\star} p<0.1$

Fuente: elaboración propia.

La proporción de la fuerza de trabajo que cuenta con educación superior, media superior y secundaria terminada tiene un impacto positivo adicional al capturado por la PEA total sobre el desempeńo económico de los estados. Sin embargo, los coeficientes asociados a dichas proporciones no son estadísticamente significativos. Bajo esta especificación, el capital humano altamente calificado parece no impactar de manera significativa al nivel de ingresos. Por otro lado, las entidades donde la proporción de la PEA con secundaria incompleta y primaria es alta, tienden a mostrar valores de producción inferiores. El modelo FE sugiere que un incremento del 10\% en el número de investigadores SNI se asocia a un efecto no significativo sobre el PIB estatal. Respecto al conocimiento intangible, el modelo FE indica que cuando se incrementa dicho conocimiento en $10 \%$, los estados muestran un aumento en los niveles de producción del orden del $0.10 \%$. Por lo tanto, la existencia de capital intangible se asocia con incrementos de magnitud limitada en el nivel de actividad económica de los estados.

El modelo FE identifica una relación negativa y significativa entre la IED y el PIB, y contradice las expectativas iniciales. Los autores argumentan que dicha relación puede deberse a la omisión de variables relevantes en la función de producción, e.g. capital natural o alguna medida adecuada de capital físico. Además de ello, los destinos de la IED podrían no estar vinculados al impulso de actividades relacionadas con la transferencia de conocimientos. Por otro lado, el grado de apertura comercial mantiene una relación positiva y significativa con el nivel de producción. El parámetro correspondiente sugiere que 
cuando el grado de apertura aumenta su intensidad en 10\%, el nivel del PIB crece en promedio $0.33 \%$, lo que sugiere que existe un canal de transmisión de conocimiento vía intercambio de mercancías y servicios adquiridos en el exterior y que dicha interacción conlleva a niveles de producción ligeramente más altos.

Con el propósito de verificar que los resultados anteriores sean consistentes, se estima un modelo GMM para incorporar efectos dinámicos de las variables explicativas. El cuadro 2 sugiere que, al incorporar dichos efectos, se observa una contribución directa y significativa de la proporción de trabajadores con educación superior y de la formación bruta de capital fijo que llevan a cabo los gobiernos estatales sobre el producto. Los resultados restantes son consistentes con las estimaciones del modelo FE. Utilizando las pruebas de correcta especificación en el cuadro 1, el cuadro 2 muestra los resultados de los modelos SARFE, SEM-FE y SAC-FE. Los resultados del modelo SAR-FE son consistentes con los resultados del modelo panel de FE. Además de las relaciones identificadas en el modelo $\mathrm{FE}$, este modelo identifica el grado de correlación espacial entre el nivel de actividad económica de estados colindantes $(\rho=0.23)$. El valor del parámetro $\rho$ indica que el crecimiento (el decrecimiento) de una entidad federativa beneficia (perjudica) a los estados vecinos. Este resultado sugiere que los proyectos de desarrollo suelen tener impactos multiplicadores, no sólo en la zona de interés, sino también en entidades colindantes.

El cuadro 2 muestra los parámetros estimados del modelo SEM-FE. En general, los coeficientes asociados a los efectos directos son consistentes con los resultados de los modelos FE y SAR-FE. La significancia estadística del parámetro asociado a la correlación espacial de los errores sugiere que existe un patrón de correlación en la heterogeneidad no observada de las entidades federativas colindantes. Dado que, por ejemplo, el monto de la formación bruta de capital fijo del sector privado o el capital natural son parte del término de error, la significancia estadística del parámetro $\lambda$ podría estar capturando la vinculación espacial de dichas variables.

Tomando en cuenta que los modelos SAR-FE y SEM-FE indican que existe cierto grado de correlación espacial entre el nivel de actividad económica y factores de producción no observables entre estados vecinos, es decir $\rho \neq 0$ y $\lambda \neq 0$, se estiman los parámetros del modelo SAC-FE. El cuadro 2 muestra que tanto el producto estatal como el término de error de los estados vecinos se encuentran vinculados. Los coeficientes asociados a los parámetros $\rho=0.52$ y $\lambda=-0.94$ son estadísticamente significativos. Asimismo, se espera que cuando la PEA y las proporciones de ella con educación superior, con educación media y con educación secundaria aumenten $10 \%$, el PIB estatal se incremente 
en $1.10,0.04,0.096$ y $0.22 \%$, respectivamente. Se observa que al aumentar en $10 \%$ la inversión del gobierno en capital fijo, se espera que el PIB se incremente en $0.11 \%$. Estos resultados confirman la hipótesis de que mayores niveles de capital humano permiten a las entidades federativas aspirar a un nivel de actividad económica superior y que, dicho dinamismo, se diluya en el espacio generando un efecto multiplicador en las regiones aledañas.

\section{DISCUSIÓN}

Los resultados de la investigación sugieren que la economía del conocimiento tiene una asociación débil con el nivel de crecimiento de las entidades federativas. A pesar de que la proporción de trabajadores con bajos niveles educativos mantiene una relación negativa con el producto, la proporción de trabajadores con alta calificación tienden a mostrar una asociación limitada o nula con el producto.

De acuerdo con la ocDe (2019a), el 82\% de los mexicanos entre 25 y 64 años no cuentan con estudios de educación superior, siendo inferior al promedio de la organización (63\%). Esto podría explicar la asociación no significativa entre el producto y la mano de obra altamente calificada. Medidas de política que promuevan la educación de la mano de obra deben acompañarse del fortalecimiento del vínculo universidad-industria. El estudio (OCDE, 2019b) señala que casi uno de cada dos egresados de educación superior tiene un empleo que no requiere su calificación, más del $25 \%$ trabaja en la economía informal y que más de la mitad de las empresas encuentran dificultades para cubrir sus vacantes. En este sentido, la desvinculación entre los objetivos de la política educativa y las necesidades del mercado laboral podrían ser un argumento para explicar la nula asociación entre calificación de la fuerza laboral y producto. Bajo las circunstancias anteriores, las partes interesadas: gobierno federal, gobiernos estatales, empresas y trabajadores podrían iniciar un diálogo para la alineación de los objetivos de la política educativa y los requerimientos de la industria.

El número de investigadores SNI se asocia con niveles de producción superiores, sin embargo, dicha relación no es significativa. Este resultado puede explicarse por la desvinculación entre centros de investigación y empresas y porque sólo 33\% de los investigadores en México pertenecen al sNI, de los cuales, una proporción menor desarrolla tecnología aplicada a los procesos productivos (FCCT, 2016). Asimismo, algunos estudios argumentan que los conocimientos generados por la investigación pueden o no utilizarse en 
los procesos productivos que se llevan a cabo en la región donde se producen o en otras regiones (Acs et al., 2002). Para comprobar la hipótesis anterior, se tendría que analizar la correlación espacial entre investigadores sNI y nivel de producto regional. Sin embargo, este fenómeno tendría que identificarse mediante el uso de información microeconómica o ampliando la cobertura espacial de este trabajo, e.g. información a nivel empresa o información para un conjunto de países.

Estudios previos encuentran una relación positiva entre el número de patentes y el nivel de producto, sin embargo, esta asociación no es significativa (Guzmán et al., 2018). En este trabajo se identifica una relación positiva y, en la mayoría de los casos, es significativa. Algunos estudios argumentan que el número de patentes generadas por residentes de una región pueden no ser representativas de la vigorosidad con la que se produce conocimiento y que el uso de dicho conocimiento no se limita a la región donde fue creado. Bajo estas circunstancias, los coeficientes mostrados en el cuadro 2 miden la asociación directa con el producto local, el cual subestima su efecto total. Para medir el impacto total de las patentes se podría incorporar la correlación espacial entre esta variable y el producto estatal.

Autores como Ros $(2013,2015)$ enfatizan la brecha en los niveles de producto (desarrollo) que existe entre el norte y el sur de México. El cuadro 3 muestra los parámetros estimados para el conjunto de variables explicativas en el cuadro 2 para ambas regiones. ${ }^{4}$ En general, los resultados son consistentes con las estimaciones del cuadro 2. En algunos casos, la producción en el norte del país tiende a mostrar una asociación directa y significativa con la proporción de trabajadores con educación superior, mientras que la proporción de trabajadores con estudios de secundaria incompleta y primaria tienden a asociarse con menores niveles de producto. Por su parte, el sur de México no muestra una relación clara entre la calificación de sus trabajadores y el producto, lo que podría indicar un grado de desvinculación más amplio entre los objetivos del programa educativo y los requerimientos del mercado laboral.

La formación bruta de capital fijo del gobierno mantiene una relación positiva y significativa con el nivel de producción en el norte del país, mientras que en el sur dicha asociación no es estadísticamente diferente de 0. Dicho resultado provee evidencia empírica sobre las aseveraciones en (Ros, 2015), donde se argumenta que se necesita una gran inversión en capital fijo en el sur del país para incentivar el nivel de producto. De acuerdo con los modelos

4 Una mayor desagregación de la información podría reducir considerablemente los grados de libertad de las estimaciones y, por ende, presentar un sesgo alto. 
GMM y SEM-FE, el número de investigadores SNI suele asociarse con niveles superiores de producción en el norte del país. La relación de las patentes con el producto no muestra una relación diferenciada clara entre el norte y el sur de México.

El cuadro 2 sugiere una relación negativa entre IED y producto a nivel estatal. Mientras que el cuadro 3 indica que dicha relación se manifiesta de manera significativa en el sur de México, en tanto en el norte no es estadísticamente diferente de 0 . Chiatchoua et al. (2016) encuentran que la IED tiende a disminuir el empleo en el sector primario y a desestabilizar el empleo en el sector terciario. En este sentido, la IED podría incidir negativamente en el producto de las entidades federativas del sur de manera indirecta a través del empleo, puesto que, las actividades agropecuarias, forestales y turísticas representan una parte importante del PIB.

Para corroborar la consistencia de los resultados se reemplaza el PIB nacional total con el piв de las industrias manufactureras. En general, los resultados son similares a los del cuadro 1 . Se confirma la desvinculación entre el número de investigadores SNI y la producción de la manufactura, una asociación débil entre el número de patentes y la producción y se observa una asociación mayor entre la intensidad de comercio con el exterior y el pIB de las manufacturas. Asimismo, los patrones de correlación espacial se mantienen. ${ }^{5}$

Algunas formas de acumulación de conocimiento podrían estar correlacionadas y, quizá, tener implicaciones sobre los resultados obtenidos. Por ejemplo, el número de investigadores pertenecientes al SNI podría asociarse directamente con un número mayor de patentes o la IED con el nivel de vinculación de la entidad federativa correspondiente con el exterior. En general, no se observan valores altos de correlación, excepto en casos de la estructura de la PEA por nivel de educación con la misma y del número de patentes e investigadores SNI. En el primer caso, se espera que las interacciones PEA-proporciones de PEA por nivel educativo estén correlacionadas de manera directa, pues, la PEA es un elemento de dichas interacciones. En el segundo caso, se observa un coeficiente de correlación de 0.60 , lo cual podría sugerir que un número mayor de investigadores resulta en un nivel de propiedad intelectual mayor y que su asociación con el producto se manifiesta a través de las patentes. Sin embargo, cuando se elimina el número de investigadores SNI del conjunto de estimaciones la significancia estadística del coeficiente asociado al número de patentes no se modifica. ${ }^{6}$

5 Estimaciones disponibles con solicitud a los autores.

6 Tabla de coeficientes de correlación parcial disponible con solicitud a los autores. 


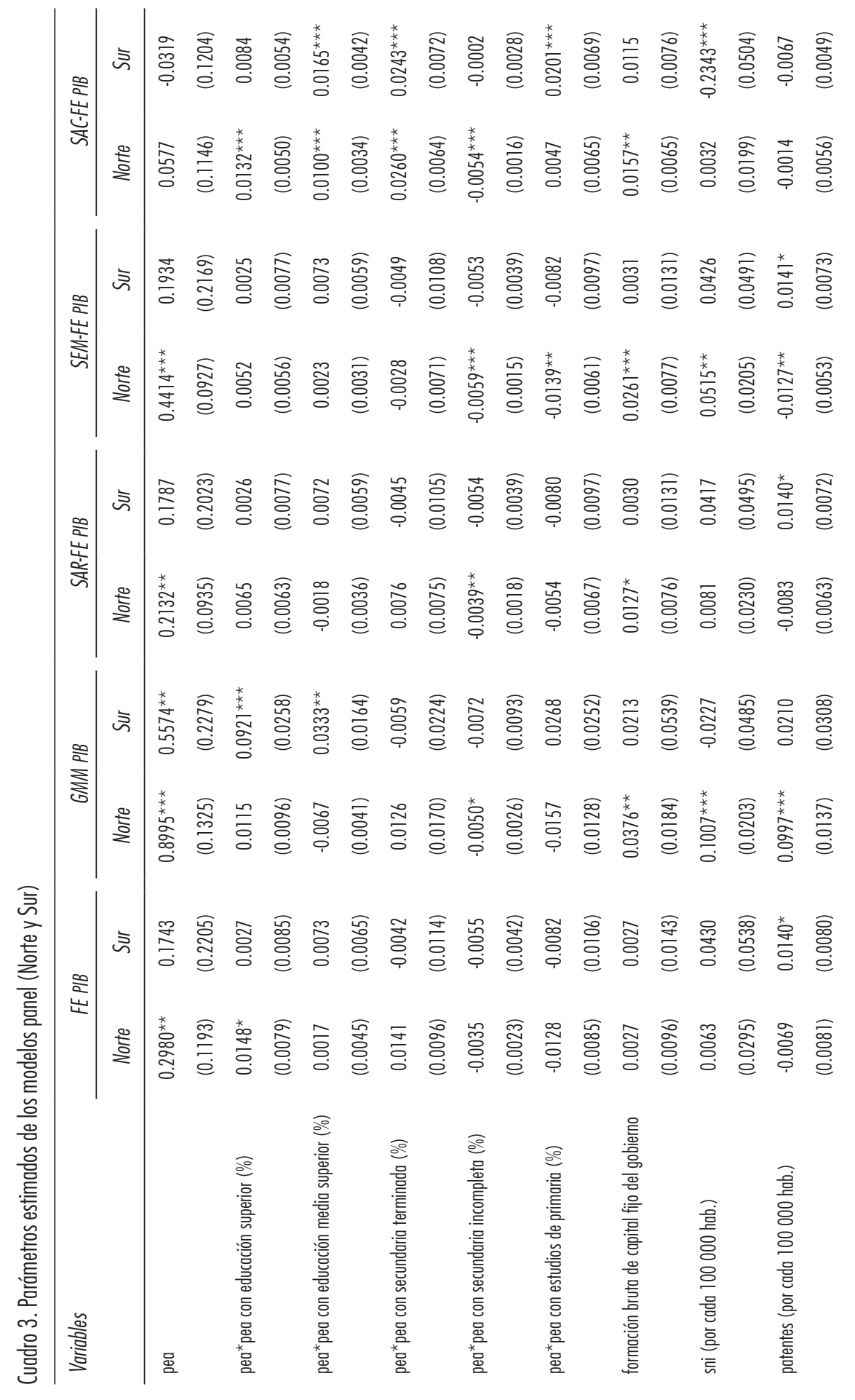




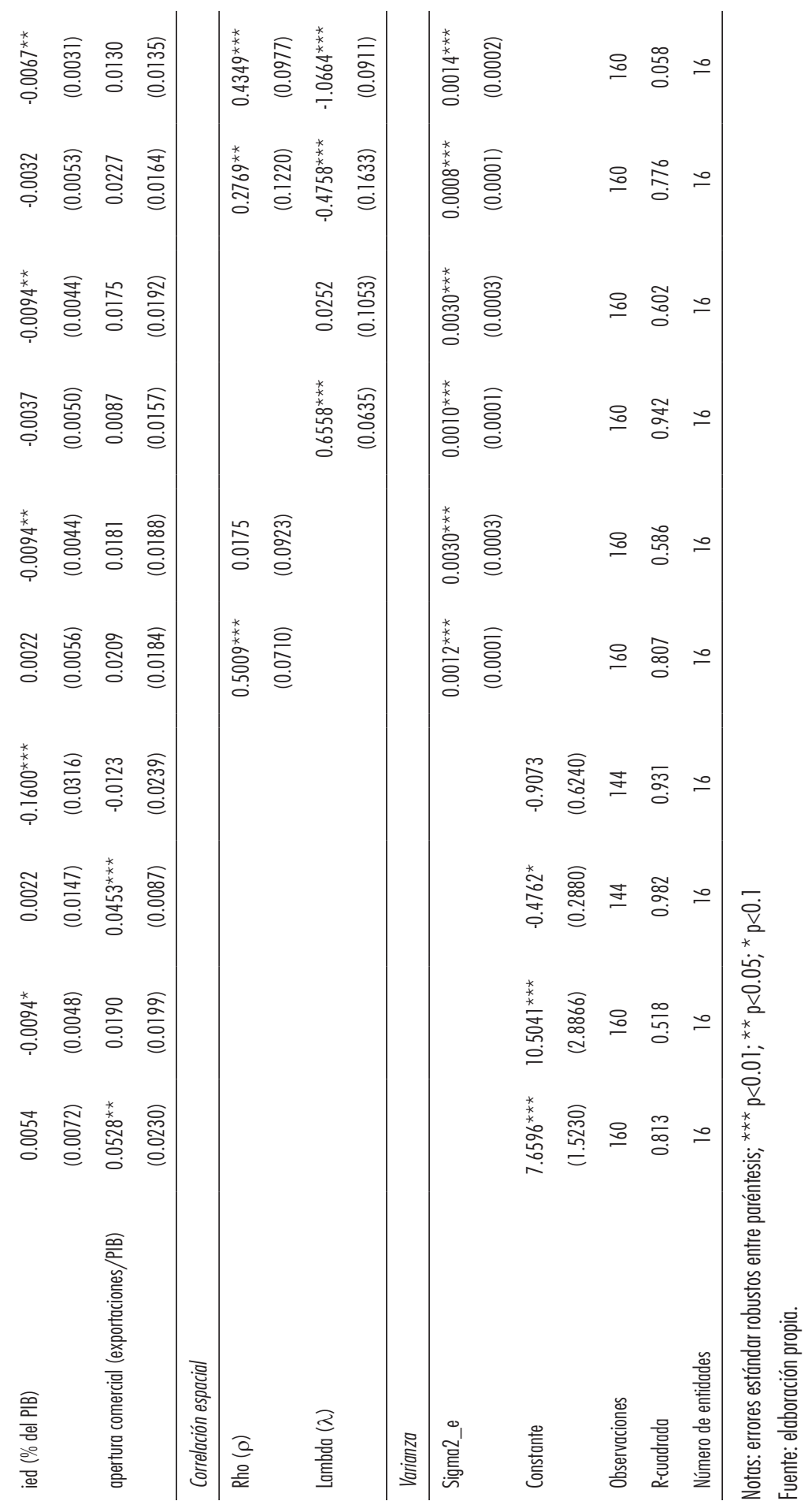


Esta investigación contribuye al debate actual sobre el fortalecimiento del vínculo universidad o centros de investigación con la industria. Los resultados sugieren que la política económica debe poner especial énfasis en fortalecer la economía del conocimiento mediante la conjunción de intereses de la sociedad, gobierno, empresas y centros de investigación.

\section{CONCLUSIONES}

Los resultados de este trabajo sugieren que las entidades federativas que cuentan con una proporción menor de trabajadores con educación básica suelen observar niveles de producción menores. El resultado confirma que niveles inferiores de capital humano limitan el crecimiento económico estatal. Asimismo, se identifica una relación débil entre proporciones de trabajadores con educación superior y nivel de producción. Lo que sugiere una desvinculación entre los objetivos de las políticas educativa e industrial.

Respecto al número de investigadores SNI, la función de producción estimada señala que de incrementarse $10 \%$ el número de investigadores se presentaría un efecto nulo sobre el pIв estatal. El Foro Consultivo Científico y Tecnológico (FCCT, 2016) señala que en el periodo 1993-2011, el 33\% del total de investigadores en México fue miembro del sNi y que una proporción menor de investigadores SNI, especialmente de las áreas de ingenierías, biotecnología y ciencias agropecuarias, se vinculan con la innovación de tecnología en el sector productivo. Esto implica que, la mayoría de los investigadores SNI producen conocimiento científico básico y muy pocos se enfocan a la producción de conocimiento tecnológico aplicado. ${ }^{7}$ Lo anterior justifica la insignificancia estadística de la asociación investigadores SNI y producción estatal.

El nivel de capital intangible muestra una asociación positiva con el nivel de producto de las entidades federativas en la mayoría de las estimaciones. Al incrementar el número de patentes $10 \%$, se espera un incremento del producto en promedio de $0.10 \%$ en el PIB estatal. El resultado anterior sugiere que la acumulación de capital intangible promueve el desarrollo económico estatal de forma limitada. Es importante resaltar que el uso de patentes no se limita a empresas domésticas, i.e. pertenecientes a la misma entidad federativa, por lo tanto, este resultado debe interpretarse tomando en cuenta

7 Véase creación del Programa de Estímulos a la Innovación (PEI). El PEI, creado por CONACYT, destina recursos económicos para incentivar a los empresarios a generar innovaciones tecnológicas con el objetivo de que dichas innovaciones impulsen el desarrollo económico en México. 
dicha limitante. Algunas entidades federativas pueden beneficiarse del stock de conocimiento generado en otra región. Desafortunadamente, la falta de información estadística a nivel macroeconómico limita el análisis detallado del uso de patentes.

Los canales de difusión de conocimiento también juegan un papel importante en el nivel de producto estatal. El grado de apertura comercial, especialmente con Estados Unidos, impacta positivamente la producción. Al incrementarse el monto de exportaciones como proporción del PIB en 10\%, respecto al nivel actual, se espera que el producto muestre un alza del $0.40 \%$. Sin embargo, los flujos de inversión extranjera directa parecen mantener un efecto negativo sobre el producto estatal, especialmente en el sur del país. Este resultado puede deberse a la omisión de variables relevantes en la función de producción, como la falta de información sobre importaciones de bienes y servicios a nivel estatal y la proporción de importaciones y exportaciones de bienes de alta tecnología, o a los impactos que tiene la IED en el nivel de empleo del sector primario y terciario en el sur de México.

Autores como Ros $(2013,2015)$ sugieren que la brecha en los niveles de producción y de desarrollo entre el norte y el sur del país se puede cerrar mediante inversiones importantes en capital físico. Cuando se diferencia entre norte y sur, en este trabajo se encuentra que en el norte existe una asociación directa entre la proporción de trabajadores con educación superior y el nivel de producto, mientras que en el sur la proporción de trabajadores con estudios como secundaria incompleta y primaria tiende a asociarse con niveles de producción más bajos. La formación bruta de capital fijo por el gobierno se asocia con niveles de producto más altos en el norte, mientras que dicha relación no es estadísticamente diferente de 0 en el sur. Dicho resultado brinda evidencia empírica adicional al argumento de Ros (2015) sobre la necesidad de incrementar la inversión en el sur de México. Asimismo, en algunos casos se encuentra que el número de investigadores SNI se asocian con mayores niveles de producción en el norte del país, lo que, sugeriría que el fortalecimiento del vínculo entre la universidad o centros de investigación y la industria representa un instrumento útil en la promoción del crecimiento económico.

En la interpretación de resultados de este trabajo, el lector deberá considerar lo siguiente: la falta de información estadística sobre el stock o flujos de capital físico con los que cuentan las entidades federativas limita el análisis. A pesar de que se tomó en cuenta la correlación espacial del término de error, el cual contiene variables como capital físico y capital natural, en los modelos panel es imposible incorporar estos factores de producción en el conjunto de estimaciones. Como extensión de este trabajo de investigación se sugiere la 
estimación de un modelo a nivel microeconómico que incorpore la heterogeneidad de los agentes económicos en el análisis.

El número de patentes y de investigadores pertenecientes al SNI por habitante podrían no ser medidas adecuadas del capital intelectual y de la capacidad de innovación en las entidades federativas, respectivamente. Se podría argumentar entonces que en México existe una desvinculación entre la acumulación de capital intelectual, la producción de nuevos conocimientos científicos y los procesos productivos, por ende, su asociación con el crecimiento económico es limitado. Asimismo, el número de investigadores sNi podría no reflejar la capacidad de innovación de algunas entidades federativas, puesto que, existen institutos y centros de investigación, especialmente privados, donde sus investigadores no pertenecen al SNI y podrían vincularse con mayor vigorosidad con el sector productivo. Por lo tanto, los resultados de este trabajo de investigación deben leerse tomando en cuenta el conjunto de limitantes mencionadas anteriormente.

\section{AGRADECIMIENTOS}

Los autores agradecen el financiamiento otorgado por el Consejo Nacional de Ciencia y Tecnología (CONACYT) mediante el proyecto titulado Modelo de Innovación basado en la Economía Nacional del Conocimiento con número 201501-1309. Así como los comentarios de árbitros anónimos que contribuyeron de manera sustancial al desarrollo de este artículo.

\section{BIBLIOGRAFÍA}

Acs, Z. J., Anselin, L. y Varga, A. (2002), "Patents and innovation count as measures of regional production of new knowledge", Research Policy, vol. 31, núm. 7, DOI <https://doi.org/10.1016/S0048-7333(01)00184-6> Anselin, L. (2013), Spatial Econometrics: Methods and Models, vol. 4, Springer Science \& Business Media, DOI <https://doi.org/10.1007/97894-015-7799-1>

Arrow, K. J. (1971), "The economic implications of learning by doing”, Readings in the Theory of Growth, London, Palgrave Macmillan.

Basant, R. y Fikkert, B. (1996), "The effects of R\&D, foreign technology purchase, and domestic and international spillovers on productivity in 
Indian firms", The Review of Economics and Statistics, DOI <https://doi. org/10.2307/2109920>

Basurto, S. y Sánchez, G. (2020), Technical efficiency in small and mediumsized firms: a stochastic frontier analysis. En proceso de publicación.

Becker, G. S. (1994), "Human capital revisited", Human capital: A theoretical and empirical analysis with special reference to education, $3^{\mathrm{a}}$ edición, Chicago, The University of Chicago Press.

Cameron, A. C. y Trivedi, P. K. (2005), Microeconometrics: Methods and applications, Cambridge, Cambridge University Press.

Chiatchoua, C., Castillo, O. N. y Santibáñez, A. L. V. (2016), "Inversión Extranjera Directa y empleo en México: análisis sectorial”, Economía Infor$m a, 398$, DOI $<10.1016 /$ j.ecin.2016.04.004>

Coe, D. T. y Helpman, E. (1995), "International R\&D spillovers", European Economic Review, vol. 39, núm. 5, DOI <https://doi.org/10.1016/00142921(94)00100-E>

, Helpman, E. y Hoffmaister, A. W. (1997), "North-south R\&D spillovers", The Economic Journal, vol. 107, núm. 440, DOI <https://doi. org/10.1111/1468-0297.00146>

Coleman, J. S. (1988), "Social capital in the creation of human capital", American Journal of Sociology, 94, S95-S120, The University of Chicago Press.

Engelbrecht, H.-J. (1997), "International R\&D spillovers, human capital and productivity in OECD economies: An empirical investigation", European Economic Review, vol. 41, núm. 8, DOI <https://doi.org/10.1016/S00142921(96)00046-3>

Foro Consultivo Científico y Tecnológico A.C. (FCCT) (2016), El Sistema Nacional de Investigadores en números. Recuperado de <http://www.foroconsultivo.org.mx/libros_editados/SNI_en_numeros.pdf>

Frantzen, D. (2000), "Innovation, international technological diffusion and the changing influence of $\mathrm{R} \& \mathrm{D}$ on productivity", Cambridge Journal of Economics, vol. 24, núm. 2, DOI <https://doi.org/10.1093/cje/24.2.193>

Guellec, D. y De La Potterie, B. v. P. (2002), "R\&D and productivity growth", OECD Economic Studies, vol. 2001, núm. 2, DOI <https://doi. org/10.1787/16097491>

Guzmán, A., Gómez Víquez, H. y López Herrera, F. (2018), "Patentes y crecimiento económico, el caso de México durante el TLCAN”, Economía: teoría y práctica (SPE4), México, DOI <http://dx.doi.org/10.24275/etypuam/ne/ e042018/guzman> 
Hausman, J. A. (1978), "Specification tests in econometrics", Econometrica: Journal of the Econometric Society, vol. 46, núm. 6, DOI <10.2307/1913827> Houghton, J. y Sheehan, P. (2000), A primer on the knowledge economy, Centre for Strategic Economic Studies, Australia, Victoria University.

Instituto Nacional de Estadística y Geografía (INEGI) (2019), Banco de Información Económica. Recuperado de <https://www.inegi.org.mx/sistemas/ bie/??

Kao, C., Chiang, M.-H. y Chen, B. (1999), "International R\&D spillovers: an application of estimation and inference in panel cointegration", Oxford Bulletin of Economics and statistics, vol. 61, núm. S1, DOI <https://doi. org/10.1111/1468-0084.0610s1691>

Lucas Jr, Romer E. (1988), "On the mechanics of economic development", Journal of Monetary Economics, vol. 22, núm. 1, DOI <https://doi. org/10.1016/0304-3932(88)90168-7>

Mendoza-Cota, J. E. (2011), "Impacto de la inversión extranjera directa en el crecimiento manufacturero en México", Problemas del Desarrollo. Revista Latinoamericana de Economía, vol. 42, núm. 167, DoI <http://dx.doi. org/10.22201/iiec.20078951e.2011.167.27720>

Mincer, J. (1958), "Investment in human capital and personal income distribution", Journal of Political Economy, vol. 66, núm. 4, The University of Chicago Press.

Naveed, A. y Ahmad, N. (2016), "Technology spillovers and international borders: a spatial econometric analysis", Journal of Borderlands Studies, vol. 31, núm. 4, DOI <https://doi.org/10.1080/08865655.2016.1188 669>

Nursamsu, S. y Hastiadi, F. F. (2015), "Analysis of international R\&D spillover from international trade and foreign direct investment channel: evidence from asian newly industrialized countries", Journal of Economic Cooperation \& Development, vol. 36, núm. 2, Turkey.

Organización para la Cooperación y Desarrollo Económicos (oECD) (2019a), "El futuro de la Educación Superior en México: promoviendo calidad y equidad". Reporte técnico. Recuperado de <https://scholar.google.es/ scholar?hl=es\&as_sdt $=0 \% 2 \mathrm{C} 5 \& \mathrm{q}=\mathrm{OECD}+\% 282019 \mathrm{a} \% 29 \% 2 \mathrm{C}+\% \mathrm{E} 2$ $\% 80 \% 9 \mathrm{CEl}++$ Futuro+de+la+Educaci $\% \mathrm{C} 3 \% \mathrm{~B} 3 \mathrm{n}+$ Superior+en+M $\% \mathrm{C} 3$ $\%$ A $9 x i c o \% 3 \mathrm{~A}+$ Promoviendo+Calidad $+\mathrm{y}+$ Equidad $\%$ E2\%80\%9D.+Rep orte $+\mathrm{t} \% \mathrm{C} 3 \% \mathrm{~A} 9 \mathrm{cnico} . \& \mathrm{btnG}=>$

(2019b), "La Educación Superior en México: resultados y relevancia para el mercado laboral”. Reporte técnico. Recuperado de <http://www. 
oecd.org/about/secretary-general/estudios-de-la-ocde-sobre-educacionsuperior-en-mexico-january-2019-sp.htm>

Romer, P. M. (1990), "Endogenous technological change", Journal of Political Economy, vol. 98, núm. 5 (parte 2), DOI <https://doi.org/10.1086/261725> Ros, J. (2013), Algunas tesis equivocadas sobre el estancamiento económico de México, México, El Colegio de México AC-unam. Disponible en $<$ https:// www.jstor.org/stable/j.ctt14jxqmz>

(2015), ¿Cómo salir de la trampa del lento crecimiento y alta desigualdad?, México, El Colegio de México ac-unam. Disponible en <https:// www.jstor.org/stable/j.ctt19qghp7>

Rosen, S. (1976), "A theory of life earnings", Journal of Political Economy, vol. 84, núm. 4 (parte 2), S45-S67, The University of Chicago Press.

Schultz, T. W. (1961), "Investment in human capital", The American Economic Review, vol. 51, núm. 1, American Economic Association.

Teece, D. J. (1998), "Capturing value from knowledge assets: The new economy, markets for know-how, and intangible assets", California Management Review, vol. 40, núm. 3, DoI <https://doi.org/10.2307/41165943>

Zhu, L. y Jeon, B. N. (2007), "International R\&D spillovers: Trade, FDI, and information technology as spillover channels", Review of International Economics, vol. 15, núm. 5, DOI <https://doi.org/10.1111/j.14679396.2007.00691.x> 
